\title{
LE PHYLUM DES ACANTHOCEPHALA
}

Troisième note

La Classe des PALAEACANTHOCEPHALA (Meyer, 1931) (suite)

\author{
Par Yves J. GOLVAN
}

\section{Diagnose de la famille des aRHYTHMACANTHIDA Yamaguti 1935}

Palaeacanthocephala; parasites de Poissons de mer. Tronc orné dans sa partie antérieure d'épines cuticulaires. Sous-cuticule épaisse, contenant des fragments de noyaux géants. Quatre cellules musculaires de grande taille, avec noyaux géants sphéroïdaux placés à hauteur de l'extrémité inférieure des lemnisci. Canaux principaux du système lacunaire latéraux unis par des anastomoses formant un réseau à mailles grossièrement polyédriques. Proboscis relativement court, cylindroïde ou en courte massue, armé d'au moins deux types de crochets. Crochets supérieurs à racines bien développées de forme simple, crochets inférieurs à racines rudimentaires. Cou court. Réceptacle assez court, à double paroi. Ganglion cérébroïde placé au fond du réceptacle. Lemnisci digitiformes toujours plus longs que le réceptacle. Organes mâles occupant au moins la moitié postérieure du tronc. Testicules sphéroïdaux, placés l'un derrière l'autre et en contact. Toujours six glandes cémentaires courtes et piriformes. Orifices génitaux terminaux dans les deux sexes. Canaux déférents larges et sinueux. Organe de Saefftigen toujours volumineux. Embryophores encore non décrits mais possédant cependant, selon toutes probabilités, la morphologie caractéristique d'un cycle aquatique.

GENRE TYPE : Arhythmacanthus Yamaguti 1935.

Cette famille comprend, en outre, le genre Heterosentis Van Cleave 1931.

\section{Diagnose DU GenRe Arhythmacanthus Yamaguti 1935}

Arhythmacanthidæ; parasites de Poissons de récif du Japon. Corps de petite taille. Tronc en court fuseau (la plus grande largeur est au niveau de la moitié de la longueur). Partie antérieure du tronc ornée 
d'un champ unique de très petites épines, très serrées les unes contre les autres et enchâssées dans un repli de la cuticule (d'où aspect écailleux de cette partie du tronc). Les épines sont plus lâchement réparties dans la partie postérieure du champ, qui couvre presque toute la moitié antérieure du tronc. Proboscis court, sphéroïdal, armé de files longitudinales de crochets de trois types : petits crochets apicaux, grands crochets moyens et petites épines basales. Cou court. Réceptacle court. Ganglion cérébroïde placé au fond du réceptacle. Lemnisci digitiformes, un peu plus longs que le réceptacle. Organes mâles occupant la moitié postérieure du tronc. Testicules sphéroïdaux, placés obliquement l'un derière l'autre et en contact. Six glandes cémentaires sphéroïdales ou en courte massue. Femelles inconnues.

EsPÈcE TYPE: Arhythmacanthus fusiformis Yamaguti 1935 (Japon).

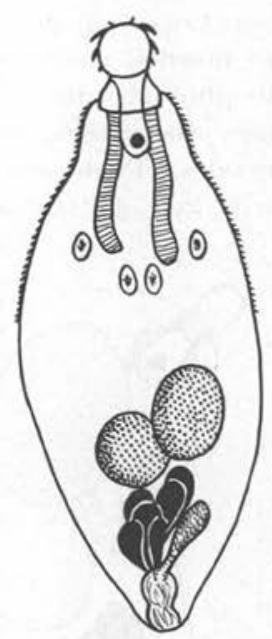

Fig. 19. - Schéma du genre Arhythmacanthus Yamaguti 1935 .

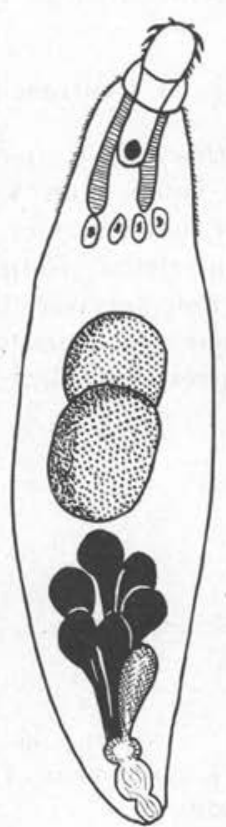

Fig. 20. - Schéma du genre $\mathrm{He}$ terosentis Van Cleave 1931.

Diagnose du GenRe Heterosentis Van Cleave 1931

Arhythmacanthidx; parasites de Poissons de mer. Tronc cylindroïde ou fusiforme, orné, dans sa partie antérieure, d'un champ unique de petites épines cuticulaires non enchâssées dans un repli de la cuticule, 
serrées les unes contre les autres dans la partie antérieure du champ, plus lâchement réparties dans la partie postérieure. Proboscis en courte massue armé de deux types de crochets: crochets vrais apicaux et épines basales. Cou assez court. Réceptacle court et large. Ganglion cérébroïde placé au fond du réceptacle. Lemnisci sacciformes, un peu plus longs que le réceptacle. Organes mâles occupant les deux tiers postérieurs du tronc. Testicules volumineux, sphéroïdaux, placés l'un derrière l'autre et largement en contact. Six glandes cémentaires courtes et piriformes, disposées en trois paires. Orifices génitaux terminaux dans les deux sexes.

EsPÈcE TYPE : Heterosentis heteracanthus (von Linstow 1896) Van Cleave 1931 (=Echinorhynchus heteracanthus v. L. 1896) (région néotropicale).

\section{$2^{\circ}$ Heterosentis plotosi Yamaguti 1935 (Japon).}

Diagnose DE LA FAMILle des HYPOECHINORHYNCHIDA nov. fam.

Palaeacanthocephala; parasites de Poissons de mer d'Extrême-Orient. Habitus très voisin d'un Neoacanthocephala. Corps de petite taille, fortement arqué selon sa face ventrale. Tronc inerme, en massue, à grosse extrémité supérieure. Cuticule épaisse. Morphologie des noyaux souscuticulaires non précisée. Canaux principaux du système lacunaire latéraux, unis par des anastomoses transversales. Proboscis sphéroïdal, inséré plus près de la face ventrale que de la face dorsale, armé de files

Fig. 21. - Schéma du genre Hypoechinorhynchus Yamaguti 1939.

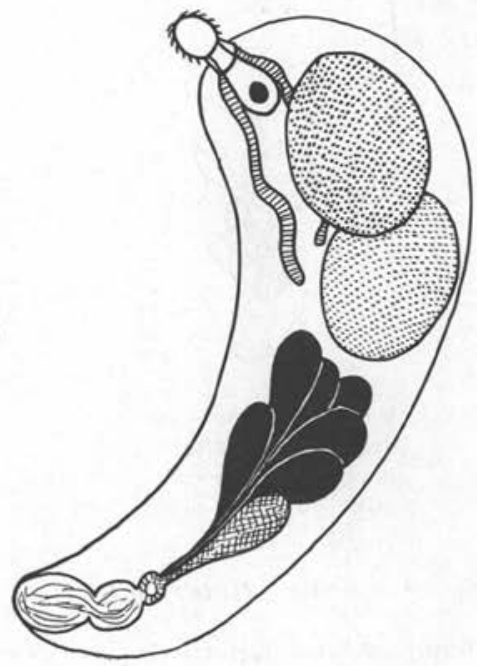


longitudinales de crochets peu nombreux. Crochets supérieurs vrais à racine bien développée, de forme simple, crochets inférieurs à racines rudimentaires (épines). Cou très court. Réceptacle à double paroi, court. Ganglion cérébroïde placé au fond du réceptacle. Lemnisci plus longs que le réceptacle, mais souvent repliés sur eux-mêmes. Rétracteurs du cou très puissants. Organes mâles occupant pratiquement toute la longueur du tronc. Testicules volumineux, sphéroïdaux, placés l'un derrière l'autre et en contact. Testicule antérieur placé dorsalement par rapport au réceptacle. Six glandes cémentaires piriformes, disposées assez régulièrement en trois paires. Orifice mâle terminal. Vulve termino-ventrale. Embryophores ellipsoïdaux, à coque mince, avec grandes hernies polaires de la membrane moyenne.

GENRE TYPE : Hypoechinorhynchus Yamaguti 1939.

\section{DiAgnose DU GENRE Hypoechinorhynchus Yamaguti 1939}

La diagnose du genre se confond, actuellement, avec celle de la famille.

ESPÈCE TYPE: Hypoechinorhynchus alaeopis Yamaguti 1939, parasite de Poissons du Japon.

Diagnose de la FAMILle des PARACANIHOCEPHALIDE nov. fam.

Palaeacanthocephala; parasites de Poissons d'eau douce de Sibérie. Corps de petite taille. Tronc fusiforme, inerme, étiré, dans sa partie antérieure, en un «faux-cou ». Proboscis toujours armé d'un petit nombre de files longitudinales de crochets peu nombreux. Embryophores à coque mince avec grandes hernies polaires de la membrane moyenne.

Genre Type : Paracanthocephalus Achmerov et DombrowskajaAchmerova 1941.

Nous avons été obligé de créer cette nouvelle famille pour classer deux genres décrits par Achmerov et Dombrowskaja-Achmerova (1941). La diagnose de ces deux genres est beaucoup trop succincte et les auteurs russes ne donnent aucun schéma permettant de se faire une idée approximative de ces Acanthocéphales. La redescription des spécimens s'impose.

Cette famille comprend les genres :

Fresnyarhynchus nom. nov.,

Paracanthocephalus Achmerov et Dombrowskaja-Achmerova 1941. 
DIAGNOSE DU GENRE Fresnyarhynchus nom. nov. (=Bolborhynchus Achmerov et Dombrowskaja-Achmerova 1941, préemployé)

Paracanthocephalidæ; décrit d'après quatre adultes immatures provenant d'un Cyprinida du Bassin de l'Amour. Corps court, avec faux-cou presque rectangulaire. Proboscis sphéroïdal, armé d'un petit nombre de crochets (cinq files de cinq), dont la taille diminue rapidement de l'apex à la base du rostre. A la base du proboscis, quatre ou cinq petits tubercules (crochets rudimentaires ?). Lemnisci longs, avec un noyau géant au niveau de leur extrémité inférieure.

EsPÈCE TYPE: Fresnyarhynchus exiguus (Achmerov et Dombrowskaja-Achmerova 1941) (Bassin du Fleuve Amour).

\section{Diagnose DU GEnRe Paracanthocephalus}

Achmerov et Dombrowskaja-Achmerova 1941

Paracanthocephalidæ; parasites de Poissons d'eau douce de Sibérie. Corps petit, trapu. Proboscis court, cylindroïde, armé de quelques files longitudinales de crochets peu nombreux, dont la taille décroît de l'apex à la base du rostre. Paríie antérieure du tronc étirée en un long «fauxcou » contenant le réceptacle et les lemnisci. Ganglion cérébroïde placé au fond du réceptacle. Lemnisci longs. Testicules ovoïdes. Six glandes cémentaires piriformes. Embryophores caractéristiques d'un cycle aquatique.

ESPÈce TYPE : Paracanthocephalus tenuirostris Achmerov et Dombrowskaja-Achmerova 1941 (Bassin du Fleuve Amour).

$2^{\circ}$ Paracanthocephalus curtus Achmerov et Dombrowskaja-Achmerova 1941 (Bassin du fleuve Amour).

\section{Diagnose de la famille des ECHINORHYNCHIDAE Cobbold 1876}

Palaeacanthocephala; parasites de Vertébrés aquatiques dont les hôtes intermédiaires sont des Crustacés Amphipodes ou Isopodes. Corps de taille moyenne. Tronc fusiforme ou cylindroïde mais jamais filiforme. Sous-cuticule mince, contenant de nombreux fragments de noyaux géants. Canaux principaux du système lacunaire latéraux unis par des anastomoses transversales. Proboscis plus ou moins cylindroïde, de taille moyenne, armé de files longitudinales de crochets toujours assez nombreux. Réceptacle à double paroi. Lemnisci assez courts, en massue à grosse extrémité inférieure ou aplatis en feuille. Toujours six glandes cémentaires courtes et piriformes ou sphéroïdales. Embryophores à coque mince, ellipsoïdaux, avec hernies polaires de la membrane moyenne. 
GENRE TYPE : Echinorhynchus O.F. Müller 1776.

Cette famille doit être divisée en deux sous-familles :

Neoacanthocephaloidinæ nov. sub. fam.

Echinorhynchinæ Travassos 1919.

\section{Diagnose de la sous-Famille des Neoacanthocephaloidinæ}

nov. sub. fam.

Echinorhynchidæ; parasites de Poissons de mer. Corps de taille petite à moyenne. Tronc fusiforme, orné, dans sa partie antérieure, d'épines cuticulaires très petites, disposées selon un champ unique, triangulaire, à pointe inférieure ventrale, et qui peut s'étendre fort loin en arrière. Présence possible d'épines génitales dans les deux sexes. Proboscis court, cylindroïde, armé d'un nombre assez réduit de crochets : crochets supérieurs à racines bien développées, épines basales à racines rudimentaires. Le passage des crochets aux épines se fait sans transition. Ganglion cérébroïde placé au fond du réceptacle. Lemnisci sensiblement aussi longs que le réceptacle. Organes mâles occupant au moins la moitié postérieure du tronc. Testicules sphérö̈daux, placés l'un derrière l'autre et en contact. Six glandes cémentaires piriformes ou ellipsoïdales disposées plus ou moins régulièrement en trois paires. Orifices génitaux terminaux dans les deux sexes. Embryophores caractéristiques d'un cycle aquatique.

GENRE TYPE : Neoacanthocephaloides Cable et Quick 1954.

Diagnose DU GEnRE Neoacanthocephaloides Cable et Quick 1954

La diagnose du genre se confond actuellement avec celle de la sousfamille.

ESPÈcE TYPE: Neoacanthocephaloides spinicaudatus Cable et Quick 1954 (Porto-Rico).

$2^{\circ}$ Neoacanthocephaloides neobythitis (Yamaguti 1939) Cable et Quick $1954[=$ Acanthocephaloides neobythitis Y. $1939=$ Psendorhadinorhynchus neobithytis (Y. 1939) Petrotschenko 1956] (Japon).

$3^{\circ}$ Neoacanthocephaloides rhinoplagusize (Yamaguti 1935) Cable et Quick 1954 (= Acanthocephaloides rhinoplagusiæ Y. 1935) (Japon).

Cette seconde espèce japonaise fut primitivement décrite comme possédant un tronc inerme. Yamaguti (1939), réétudiant son matériel de 1935, découvre les épines cuticulaires et l'indique dans son second travail tout en laissant, néanmoins, ces deux espèces dans le genre Acanthocephaloides. R.-Ph. Dollfus (1951) s'élève contre cette assignation et Cable et Quick (1954) créent le genre Neoacanthocephaloides, qui permet de sépa- 
rer des Acanthocephaloides sensu stricto, toujours inermes, ces espèces anatomiquement très voisines mais possédant des épines cuticulaires antérieures et même des épines génitales.

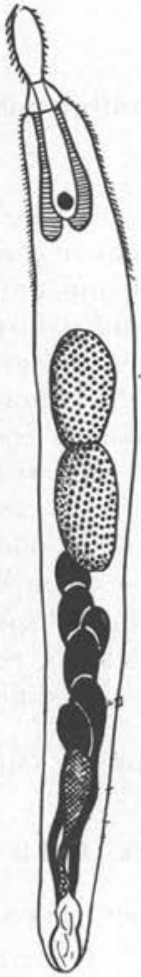

FIG. 22. - Sché$\mathrm{ma}$ du genre Neoacanthocephaloides Cable et Quick 1954 .

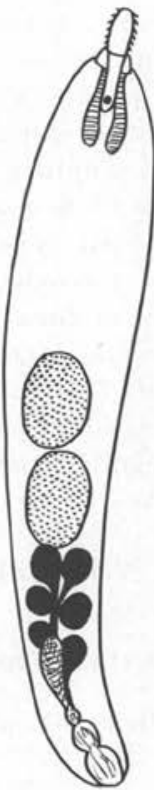

FIG, 23, - Schéma du genre Acanthocephalus Koelreuther 1771 .

Diagnose de la sous-Famille des Echinorhynchinæ Travassos 1919

Echinorhynchidæ; parasites de Poissons ou de Batraciens. Corps de taille petite à moyenne. Tronc toujours inerme, fusiforme ou cylindroïde, mais épais. Proboscis court, armé de files longitudinales de crochets, dont la taille diminue progressivement ou brusquement de l'apex à la base du rostre. Ganglion cérébroïde jamais placé dans le tiers antérieur du réceptacle. Lemnisci égaux ou à peine plus longs que le réceptacle s'ils sont digitiformes, nettement plus courts que le réceptacle s'ils sont aplatis en feuille. Six glandes cémentaires courtes, piriformes ou sphéroïdales. Embryophores caractéristiques d'un cycle aquatique. 
GENRE TYPE : Echinorhynchus O.F. Müller 1776.

Cette sous-famille comprend, en outre, les genres :

Acanthocephalus Koelreuther 1771,

Acanthocephaloides Meyer 1932.

\section{Diagnose dU GENRE Acanthocephalus Koelreuther 1771}

Echinorhynchinæ; parasites de Poissons ou de Batraciens. Tronc fusiforme, inerme. Cuticule assez épaisse. Sous-cuticule contenant de nombreux fragments de noyaux géants. Proboscis en massue plus ou moins longue, armé de crochets toujours peu nombreux, disposés selon des files longitudinales. La taille des crochets diminue toujours très progressivement de l'apex à la base du rostre, les crochets basaux étant des épines à racines rudimentaires. Ganglion cérébroïde placé au fond du réceptacle. Lemnisci sacciformes, aussi longs ou plus longs que le réceptacle. Organes mâles occupant la moitié postérieure du tronc. Testicules sphéroïdaux, placés l'un derrière l'autre, en contact ou un peu séparés. Six glandes cémentaires courtes et piriformes groupées souvent régulièrement en trois paires. Utérus court. Orifices génitaux terminaux dans les deux sexes. Embryophores ellipsoïdaux à coque mince avec grandes hernies polaires de la membrane moyenne.

EsPÈCE TYPE : Acanthocephalus anguillæ (O.F. Müller 1780) Lühe 1911 (—Echinorhynchus anguillæ O.F. M. $1780=E$. globulosus Rudolphi 1819 p.p. = Echinorhynchus linstowi Hamann $1892=$ E. proteus Porta $1905=E$. propinquus Mühling 1898), parasite de Poissons d'eau douce de l'Hémisphère Nord, dont l'hôte intermédiaire est un Isopode.

$2^{\circ}$ Acanthocephalus paronai (Condorelli 1897), qui n'est probablement qu'un synonyme d'A. anguill $x$.

$3^{\circ}$ Acanthocephalus lucii (O.F. Müller 1776) Lühe 1911 (= Echinorhynchus lucii O.F. M. $1176=$ E. angustatus Rudolphi 1809), parasite de Poissons d'eau douce et de Poissons d'eau saumâtre de la Baltique, dont l'hôte intermédiaire est un Isopode.

$4^{\circ}$ Acanthocephalus graciliacanthus Meyer 1932, parasite de Poissons d'eau douce d'Allemagne (Poméranie).

$5^{\circ}$ Acanthocephalus ranæ (Schrank 1788) Lühe 1911 (= Echinorhynchus ranæ S. $1788=$ E. haeruca Rudolphi 1809), parasite de Batraciens Anoures et Urodèles dont l'hôte intermédiaire est un Isopode (Ancien Monde).

$6^{\circ}$ Acanthocephalus falcatus (Froelich 1789) Lühe 1911, qui n'est probablement qu'un synonyme d'A. ranæ. 
$7^{\circ}$ Acanthocephalus anthuris (Dujardin 1845) Lühe 1911, parasite de Tritons et de Tortues (?) de France et d'Italie.

$8^{\circ}$ Acanthocephalus bufonis (Shipley 1903) Southwell et Macfie 1925 (= A. sinensis Van Cleave 1937), parasite de Batraciens anoures d'Extrême-Orient.

$9^{\circ}$ Acanthocephalus lucidus Van Cleave 1925 (= A. artatus Van Cleave 1925), parasite de Batraciens anoures d'Extrême-Orient.

$10^{\circ}$ Acanthocephalus nanus Van Cleave 1925, parasite de Batraciens Anoures d'Extrême-Orient.

$1^{\circ}$ Acanthocephalus gotoi Van Cleave 1925, parasite de Poissons d'Extrême-Orient.

$12^{\circ}$ Acanthocephalus echigoensis Fujita 1920 (= A. oncorhynchi Fujita $1921=A$. aculeatus Van Cleave $1931=A$. acerbus Van Cleave 1931), provenant d'Extrême-Orient et parasite de Poissons.

$13^{\circ}$ Acanthocephalus hastæ Baylis 1944 (= Echinorhynchus truttæ Schrank in Southwell et Macfie 1925 = Echinorhynchus clavula Dujardin, in Southwell et Macfie 1925), parasite de Poissons de mer du Pacifique Sud.

$14^{\circ}$ Acanthocephalus kashmiriensis Datta 1936, parasite de Poissons de l'Inde du Nord.

$15^{\circ}$ Acanthocephalus vancleavei Hughes et Moore 1943, parasite de Batraciens d'Amérique du Nord.

$16^{\circ}$ Acanthocephalus parallelotestis Achmerov et Dombrowskaja-Achmerova 1941, parasite de Poissons d'eau douce de Sibérie Orientale.

$17^{\circ}$ Acanthocephalus elongatus Van Cleave 1937, parasite de Batraciens Anoures de Chine.

$18^{\circ}$ Acanthocephalus lutzi (Von Linstow 1896), parasite de Batraciens Anoures du Brésil. L'appartenance au genre Acanthocephalus est incertaine, car la description de Linstow est rudimentaire, et Meyer, dans son dessin original, indique qu'il y a quatre et non six glandes cémentaires. De plus, l'embryophore ne présente pas de hernies polaires de la membrane moyenne et la coque est sculptée.

$19^{\circ}$ Acanthocephalus dirus (Van Cleave 1931) Van Cleave et Townsend 1936 (Echinorhynchus dirus Van Cleave 1931), parasite de Poissons d'eau douce d'Amérique du Nord.

$20^{\circ}$ Acanthocephalus acutulus Van Cleave 1931, parasite de Batraciens Urodèles d'Amérique du Nord.

$21^{\circ}$ Acanthocephalus opsariichthydis Yamaguti 1935, parasite de Poisson d'eau douce du Japon.

$22^{\circ}$ Acanthocephalus tumescens (Von Linstow 1896) Porta 1905, parasite de Poissons de la Terre de Feu.

$23^{\circ}$ Acanthocephalus amuriensis Kostylew 1941, parasite de Poissons d'eau douce du Bassin de l'Amour. 
$24^{\circ}$ Acanthocephalus japonicus (Fukui et Morishita 1936) Petrotschenko $1956[=$ Filisoma japonicum F. et M. 1936 = Acanthocephaloides japonicus (F. et M. 1936) Yamaguti 1939], parasite de Poissons de mer du Japon.

$25^{\circ}$ Acanthocephalus minor Yamaguti 1935, parasite de Poissons d'eau douce du Japon.

$26^{\circ}$ Acanthocephalus lateralis (Leidy 1851) Petrotschenko 1956, parasite de Poissons d'eau douce d'Amérique du Nord. Il s'agit, en fait, d'une species inquirenda.

\section{Diagnose DU GenRe Acanthocephaloides Meyer 1932}

Echinorhynchinæ; parasites de Poissons de mer, dont les hôtes intermédiaires sont des Crustacés. Corps de petite taille. Tronc inerme, fusiforme. Sous-cuticule contenant de très nombreux et très petits fragments de noyaux géants. Canaux principaux du système lacunaire latéraux unis par des anastomoses en réseau à larges mailles polyédriques. Cuticule mince. Proboscis court, cylindrique, armé d'un petit nombre de crochets disposés selon des files longitudinales. Crochets supérieurs de grande taille à racines bien développées. Crochets basaux petits, à racines rudimentaires. Le passage des crochets vrais aux épines se fait sans transition. Réceptacle à double paroi inséré à la base du proboscis. Ganglion cérébroïde placé au fond du réceptacle. Lemnisci aussi longs ou plus longs que le réceptacle, digitiformes. Organes mâles occupant la moitié ou moins de la moitié postérieure du tronc. Testicules sphéroïdaux, placés l'un derrière l'autre et en contact. Six glandes cémentaires sphéroïdales plus ou moins régulièrement disposées par paires ou en deux groupes de trois. Utérus long. Orifices génitaux terminaux dans les deux sexes. Embryophores ellipsoïdaux à coque mince avec hernies polaires de la membrane moyenne.

ESPÈcE TYPE : Acanthocephaloides propinquus (Dujardin 1845) Meyer 1932 (=Echinorhynchus propinquus D. $1845=E$. fabri Rudolphi $1819=E$. globulosus Rudolphi 1819 p.p. $=E$. pumilio Rudolphi 1819), parasite de Poissons de la Méditerranée et de l'Atlantique.

$2^{\circ}$ Acanthocephaloides incrassatus (Molin 1858) Meyer $1932[=$ Echinorhynchus incrassatus M. $1858=$ Echinorhynchus incrassatus in Linton $1888=E$. devisianii Molin $1858=E$. flavus Molin $1858=$ Acanthocephalus incrassatus (Molin 1861) in Kostylew 1926], parasite de Poissons de la Méditerranée et de la Mer Noire.

$3^{\circ}$ Acanthocephaloides chabanaudi Dollfus 1951, parasite de Poissons de mer des côtes Est et Ouest de l'Afrique.

$4^{\circ}$ Acanthocephaloides soleæ (Porta 1905) Petrotschenko 1956, espèce 
très insuffisamment décrite et peut-être synonyme de la suivante, parasite de Poissons de la Méditerranée.

$5^{\circ}$ Acanthocephaloides kostylewi Meyer $1932[=$ Echinorhynchus propinquus (Dujardin) in Kostylew 1926], parasite de Poissons de la Mer Noire.

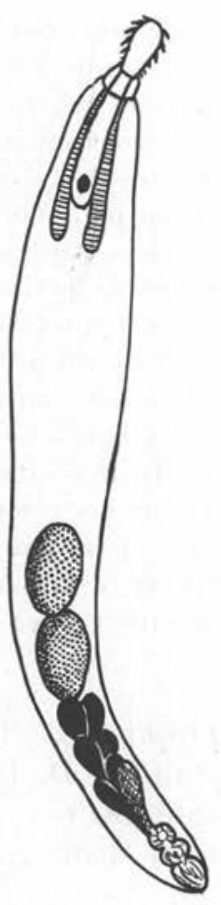

FIG. 24. - Schéma du genre Acanthocephaloides $\mathrm{M}$ e $\mathrm{y}$ e $\mathrm{r}$ 1932.

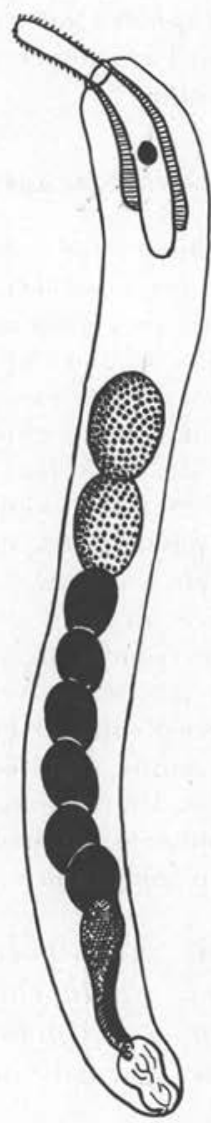

FIG. 25. - Schéma du genre Echinorhynchus o. F. Müller 1776, sous-genre Echinorhynchus.

\section{Diagnose du GenRe Echinorhynchus O.F. Müller 1776}

Echinorhynchinæ; parasites de Poissons de mer ou d'eau douce, dont les hôtes intermédiaires sont des Crustacés (Amphipodes). Corps de taille 
petite à moyenne. Tronc cylindroïde, assez épais, parfois dilaté dans sa partie antérieure, à cuticule assez épaisse, inerme. Sous-cuticule contenant de nombreux fragments de noyaux géants. Proboscis relativement long, portant des files longitudinales de crochets dont la taille décroît très progressivement de l'apex à la base du rostre et sans dissymétrie dorso-ventrale appréciable. Réceptacle inséré à la base du proboscis, à double paroi. Ganglion cérébroïde jamais placé au fond du réceptacle. Lemnisci digitiformes plus longs que le réceptacle ou aplatis en feuille et alors plus courts que le réceptacle. Organes génitaux mâles occupant les deux tiers postérieurs de la longueur du tronc. Six glandes cémentaires courtes, sphéroïdales. Orifices génitaux terminaux dans les deux sexes. Embryophores ellipsoïdaux, à coque mince, avec hernies polaires de la membrane moyenne.

EsPÈCE TYPE : Echinorhynchus gadi O. F. Müller 1776.

Petrotschenko (1956) a divisé ce genre en trois genres selon la disposition des glandes cémentaires. Nous préférons, quant à nous, garder intact le genre et réduire au rang de sous-genres les divisions de Petrotschenko.

Le genre se divise donc en trois sous-genres:

Echinorhynchus nov. sub. gen.,

Pseudoechinorhynchus (Petrotschenko 1956),

Metechinorhynchus (Petrotschenko 1956).

\section{DiAgNoSE DU SOUS-GENRE Echinorhynchus nov. sub. gen.}

Echinorhynchus; parasites de Poissons de mer, dont les six glandes cémentaires sont disposées en une seule file, les unes au-dessous des autres.

ESPÈCE TYPE : Echinorhynchus (Echinorhynchus) gadi (Zoega) in O. F. Müller $1776(=E$. acus Rudolphi $1802=E$. socialis Leidy 1856 p.p. $=E$. hepaticola von Linstow $1901=E$. arcticus von Linstow 1901).

$2^{\circ}$ Echinorhynchus (Echinorhynchus) lotellæ Yamaguti 1939, parasite de Poissons de mer du Japon.

$3^{\circ}$ Echinorhynchus (Echinorhynchus) dissimilis Yamaguti 1935, parasite de Poissons de mer du Japon.

$4^{\circ}$ Echinorhynchus (Echinorhynchus) leidyi Van Cleave 1924 (=E. salvelini Linkins 1918 in Ward et Whipple 1918), parasite de Salmonidæ migrateurs d'Amérique du Nord.

$5^{\circ}$ Echinorhynchus (Echinorhynchus) cotti Yamaguti 1935, parasite de Poisson de mer du Japon. 
$6^{\circ}$ Echinorhynchus (Echinorhynchus) attenuatus Linton 1888, parasite de Poissons de mer ou d'eau saumâtre d'Amérique du Nord.

$7^{\circ}$ Echinorhynchus (Echinorhynchus) cherchiæ Monticelli 1889, parasite d'un Poisson de mer d'Amérique Centrale.

$8^{\circ}$ Echinorhynchus (Echinorhynchus) cestodicola Von Linstow 1905, qui aurait été trouvé dans le corps même d'un Pseudophyllidæ, parasite de l'intestin d'un Poisson de mer.

$9^{\circ}$ Echinorhynchus (Echinorhynchus) abyssicola Dollfus 1931, parasite d'un Zoarcidæ abyssal de la Méditerranée.

\section{Diagnose du sous-Genre Pseudoechinorhynchus Petrotschenko} 1956.

Echinorhynchus; parasites de Poissons d'eau douce, dont les six glandes cémentaires sont disposées régulièrement en trois paires placées exactement les unes au-dessous des autres.

EsPÈcE TYPE : Echinorhynchus (Pseudoechinorhynchus) clavula Dujardin 1845 (= Echinorhynchus borealis von Linstow), parasite de Poissons d'eau douce d'Europe et probablement d'Asie.

$2^{\circ}$ Echinorhynchus (Pseudoechinorhynchus) cinctulus Porta 1905, probablement synonyme de la précédente.

$3^{\circ}$ Echinorhynchus (Pseudoechinorhynchus) parasiluri Fukui 1929, parasite de Poissons d'eau douce du Japon.

$4^{\circ}$ Echinorhynchus (Pseudoechinorhynchus) lenok Achmerov et Dombrowskaja-Achmerova 1941, parasite de Poissons du bassin de l'Amour.

\section{Diagnose du sous-Genre Metechinorhynchus Petrotschenko 1956.}

Echinorhynchus; parasites de Poissons d'eau douce, dont les six glandes cémentaires sont disposées régulièrement par paire pour les deux supérieures, alors que les quatre inférieures forment un amas compact où les glandes sont difficiles à distinguer les unes des autres.

EsPĖCE TYPE : Echinorhynchus (Metechinorhynchus) salmonis O. F. Müller 1776 (= Echinorhynchus pachysomus Creplin $1839=$ $E$. phoenyx Schneider $1903=E$. inflatus Rudolphi $1809=E$. coregoni Linkins 1918), parasite de Poissons d'eau douce de l'Hémisphère Nord, dont l'hôte intermédiaire est un Amphipode.

$2^{\circ}$ Echinorhynchus (Metechinorhynchus) truttæ Schrank $1788[=E$. fusiformis Rudolphi $1809=E$. clavula Hamann $1892=E$. clavula (Dujardin in Von Linstow 1895)], parasite de Poissons d'eau douce de 
FIG. 26. - Schéma du genre Echinorhynchus o. F. Müller 1776 , sous-genre Pseudechinorhynchus Petrotschenko 1956.
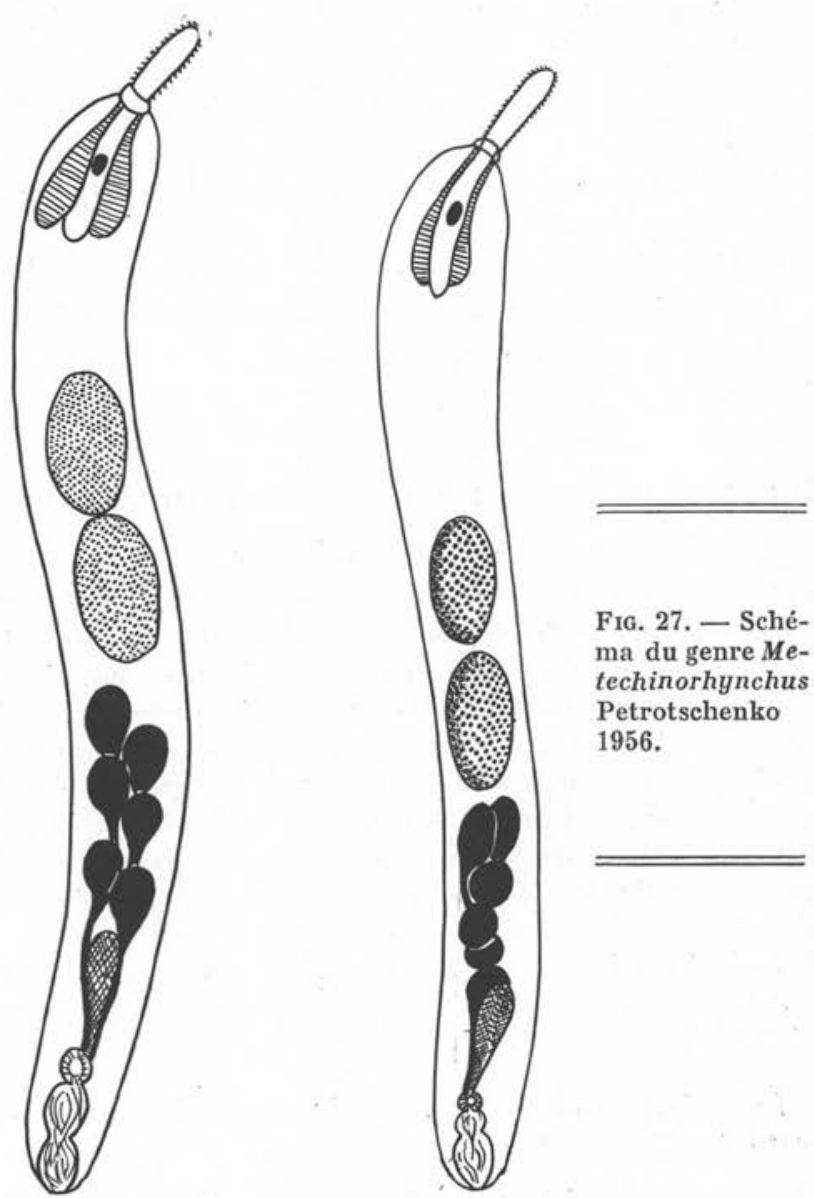

l'Hémisphère Nord. Ce parasite aurait été trouvé chez des Poissons de mer d'Australie (Johnston et Deland 1929), mais il s'agit certainement d'une erreur de détermination.

$3^{\circ}$ Echinorhynchus (Metechinorhynchus) baeri Kostylew 1928, parasite de Salmonidæ d'Arménie.

$4^{\circ}$ Echinorhynchus (Metechinorhynchus) sevani Dinnik 1933, parasite d'un Salmonidre d'Arménie. Cette espèce est peut-être synonyme de la précédente. L'hôte intermédiaire est un Amphipode.

$5^{\circ}$ Echinorhynchus (Metechinorhynchus) alpinus Von Linstow 1901, parasite de Poissons d'eau douce d'Asie.

$6^{\circ}$ Echinorhynchus (Metechinorhynchus) jucundus Travassos 1923, parasite d'un Poisson d'eau douce du Brésil. 
Echinorhynchus, qu'il nous est impossible de répartir dans l'un des trois sous-genres :

Echinorhynchus orientalis Kaw 1951 (Indes), décrit d'après une seule femelle.

Echinorhynchus gracilis Machado Filho 1948 (Brésil), décrit seulement d'après des femelles.

Echinorhynchus salobrensis Machado Filho 1948 (Brésil).

Echinorhynchus gomesi Machado Filho 1948 (Brésil).

Nous n'avons pu nous procurer l'article original de Machado Filho (1948).

\section{Diagnose de la famille des CAVISOMIDAE (Meyer 1932)}

Palaeacanthocephala; parasites, à l'état adulte, du tube digestif de Poissons d'eau douce ou de Poissons de mer. Corps de taille petite à moyenne. Tronc fusiforme ou cylindroïde, légèrement dilaté dans sa partie antérieure. Epines cuticulaires absentes ou présentes. Sous-cuticule contenant des noyaux géants fragmentés. Canaux principaux du système lacunaire latéraux unis par des anastomoses transversales. Proboscis généralement court, armé d'un petit nombre de files longitudinales de crochets peu nombreux dont la taille diminue très progressivement de l'apex à la base du rostre et ne présentant jamais de dissymétrie dorsoventrale nette. Réceptacle inséré à la base du proboscis, à double paroi. Ganglion cérébroïde jamais placé dans la partie antérieure du réceptacle. Lemnisci de longueur variable. Toujours quatre glandes cémentaires assez courtes, piriformes ou linguiformes. Embryophores caractéristiques d'un cycle aquatique.

La présence ou l'absence d'épines cuticulaires permet de diviser cette famille en deux sous-familles:

sous-famille des Vancleaveinæ nov. sub. fam., sous-famille des Cavisominæ Meyer 1932.

\section{Diagnose de la sous-famille des Vancleaveinæ nov. sub. fam.}

Cavisomida; parasites du tube digestif de Poissons de mer ou d'eau douce d'Extrême-Orient ou du Pacifique Sud. Corps de petite taille, fusiforme. Partie antérieure du tronc ornée d'épines cuticulaires. Proboscis court, en massue ou cylindroïde. Organes mâles occupant les deux tiers postérieurs de la longueur du tronc. Quatre glandes cémentaires courtes et piriformes.

Genre type : Cleaveius Subramanian 1927.

Cette sous-famille comprend, en outre, le genre Micracanthorhynchina Strand 1936. 


\section{Diagnose DU GenRe Cleaveius Subramanian 1927}

Vancleaveinæ; parasites, à l'état adulte, de Poissons d'eau douce d'Asie. Corps de petite taille. Tronc fusiforme, la partie antérieure dilatée porte un champ d'épines cuticulaires dont la disposition diffère selon le sexe. Chez les femelles ces épines sont irrégulièrement disposées dans la partie supérieure du champ alors qu'elles sont régulièrement disposées en quinconce, selon des couronnes complètes, dans la partie inférieure. Chez les mâles, seules existent ces épines régulièrement disposées. Proboscis très court, cylindroïde, armé d'un petit nombre de crochets sans dissymétrie dorso-ventrale. Position du ganglion cérébroïde non précisée. Lemnisci très longs. Organes mâles occupant les deux tiers postérieurs de la longueur du tronc. Testicules ellipsoïdaux, placés l'un derrière l'autre et en contact. Quatre glandes cémentaires courtes et piriformes. Embryophores non décrits.

EsPÈCE TYPE : Cleaveius circumspinifer Subramanian 1927, parasite d'un Poisson d'eau douce indéterminé de Birmanie.

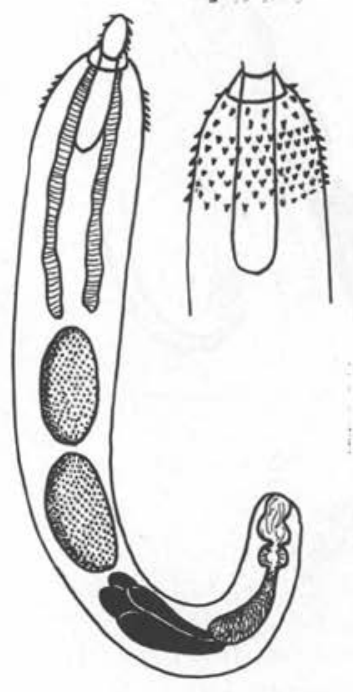

Fig. 28. - Schéma du genre Cleaveius Subramanian 1927.

DIAGNOSE DU GENRE Micracathorhynchina Strand 1936

(= Micracanthorhynchus Harada 1935

= Micracanthocephalus Harada 1938)

Vancleaveinæ; parasites, à l'état adulte, de Poissons d'eau douce d'Extrême-Orient ou de Poissons côtiers du Pacifique Sud. Corps de petite taille. Tronc fusiforme, dont la partie antérieure dilatée porte un seul 
champ d'épines cuticulaires disposées en quinconce sur une surface triangulaire à pointe inférieure ventrale. Proboscis en courte massue armé de crochets sans dissymétrie dorso-ventrale nette dont la taille diminue progressivement de l'apex à la base du rostre. Les crochets supérieurs possèdent des racines bien développées de forme simple. Les crochets inférieurs sont des épines à racines rudimentaires. Réceptacle à double paroi. Ganglion cérébroïde placé à la partie moyenne du réceptacle. Lemnisci plus courts que le réceptacle, aplatis, sacciformes. Organes mâles occupant les deux tiers postérieurs de la longueur du tronc. Testicules sphéroïdaux placés l'un derrière l'autre et en contact. Quatre glandes cémentaires courtes et piriformes. Orifice mâle un peu dorsal. Vulve ventrale avec souvent un appendice sus-vulvaire replié contre la face ventrale. Embryophores ellipsoïdes, coque mince, avec hernies polaires de la membrane moyenne.

FIG. 29. - Schéma du genre Micracanthorhynchina Strand 1936.

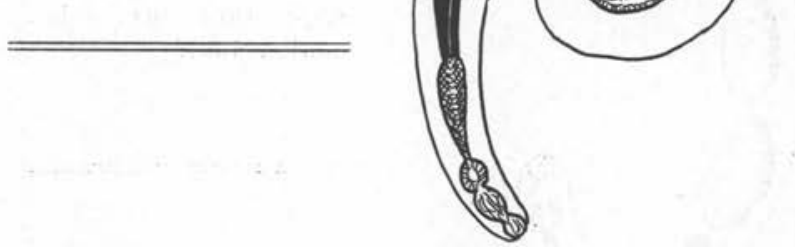

ESPÈCE TYPE : Micracanthorhynchina motomurai (Harada 1935) Ward 1951 (= Micracanthorhinnchus motomurai Harada $1935=$ Micracanthocephalus motomurai (Harada 1935) Harada 1938), provenant de Corée.

$2^{\circ}$ Micracanthorhynchina dakusuiensis (Harada 1938) Ward 1951 (= Micracanthocephalus dakusuiensis Harada 1938), provenant de Formose. Harada donne six comme nombre de glandes cémentaires pour cette espèce. Van Cleave et Lincicome (1940) placent ce genre parmi les Gorgorhynchida, puisque la description du génotype indique qu'il y a 
seulement quatre glandes cémentaires. A propos de $M$. dakusuiensis, ils écrivent : «The present writers in reviewing the Gorgorhynchidæ and the Rhadinorhynchida have found in each family complete agreement in the number of cement glands present in the representatives of all the genera. Consequently we question either the generic assignment of M. dakusuiensis or the correctness of Harada's observation that this species has 6 cement glands. 》

$3^{\circ}$ Micracanthorhynchina hemirhamphi (Baylis 1944) Ward 1951 (= Micra canthocephalus hemirhamphi Baylis 1944), parasite de Poissons côtiers de Nouvelle-Zélande et d'Australie.

\section{Diagose de la sous-Famille des Cavisominæ Meyer 1932}

Cavisomidæ; parasites du tube digestif de Poissons. Corps de taille petite à moyenne. Tronc fusiforme ou cylindroïde toujours inerme. Proboscis cylindroïde ou en massue armé de files de crochets dont la taille diminue progressivement de l'apex à la base du rostre. Réceptacle à double paroi inséré à la base du rostre. Ganglion placé dans le tiers moyen ou le tiers inférieur du réceptacle. Testicules placés l'un derrière l'autre et en contact. Quatre glandes cémentaires assez courtes, piriformes à linguiformes. Embryophores à coque mince ellipsoïdes avec grandes hernies polaires de la membrane moyenne.

GENRE TyPE : Cavisoma Van Cleave 1931.

La sous-famille comprend en outre le genre Echinorhynchoides Achmerov et Dombrowskaja-Achmerova 1941.

\section{Diagnose du genRe Cavisoma Van Cleave 1931}

Cavisominæ ; parasites, à l'état adulte, du tube digestif des Poissons de mer. Corps de taille petite à moyenne. Tronc inerme, fusiforme. Proboscis en courte massue armé de files longitudinales de crochets dont la taille diminue graduellement de l'apex à la base du rostre. Réceptacle à double paroi inséré à la base du proboscis. Ganglion cérébroïde placé à la partie moyenne du réceptacle. Lemnisci de longueur variable; plus courts ou plus longs que le réceptacle, parfois inégaux chez le même individu, digitiformes. Organes mâles occupant une portion fort variable de la longueur du tronc, allant du cinquième postérieur aux deux tiers postérieurs. Testicules ovoïdes, placés l'un derrière l'autre et en contact. Quatre glandes cémentaires assez courtes, linguiformes ou tubulaires. Embryophores ellipsoïdaux à coque mince avec grandes hernies polaires de la membrane moyenne.

EsPÈce TYPE : Cavisoma magnum (Southwell 1927) Van Cleave 1931 (= Oligoterorhynchus magnus Southwell 1927). 

Rico.

$2^{\circ}$ Cavisoma chromitidis Cable et Quick 1954, provenant de Porto-

\section{DIAGNOSE DU GENRE Echinorhynchoides}

Achmerov et Dombrowskaja-Achmerova 1941

Cavisominæ; parasites, à l'état adulte, du tube digestif des Poissons d'eau douce de Sibérie Orientale. Corps de petite taille. Tronc inerme, cylindroïde. Proboscis relativement long, cylindroïde, armé de files longitudinales de crochets dont la taille diminue très progressivement de haut en bas du rostre. Cou court. Réceptacle à double paroi. Ganglion cérébroïde placé au fond du réceptacle. Lemnisci plus courts que le réceptacle. Organes mâles dans la moitié postérieure du tronc. Testicules ovoïdes placés l'un derrière l'autre et en contact. Quatre glandes cémentaires en « épingle » (?).

ESPÈcE TYPE : Echinorhynchoides dogieli Achmerov et Dombrowskaja-Achmerova 1941.

\section{Diagnose de la famille des FISSISENTIDAE Van Cleave 1931}

Palaeacanthocephala; parasites du tube digestif de Poissons d'eau douce d'Amérique du Nord. Corps de taille assez grande. Tronc très allongé, très mince, cylindroïde, parfois un peu dilaté dans sa partie postérieure, inerme. Proboscis court, claviforme, armé de nombreux petits crochets disposés selon des files longitudinales. Réceptacle à double paroi, plus épaisse dans la partie inférieure, inséré à la base du proboscis. Ganglion cérébroïde probablement placé à la base du réceptacle. Lemnisci deux fois plus longs que le réceptacle. Organes mâles occupant les trois quarts postérieurs de la longueur du tronc. Testicules très allongés, cylindroïdes, minces, placés l'un derrière l'autre et faiblement en contact. Quatre glandes cémentaires en massue longue. Présence de deux longs diverticules à la base de la cloche utérine. Embryophores ellipsoïdaux à coque mince avec hernies polaires de la membrane moyenne.

GENRE TYPE : Fissisentis Van Cleave 1931, dont la diagnose se confond avec celle de la famille.

EsPÈcE TYPE : Fissisentis fessus Van Cleave 1931, parasite de Poissons du Mississipi.

\section{Diagnose de LA FAMILLE DES POMPHORHYNCHIDAE Yamaguti 1939}

Palaeacanthocephala; parasites de Poissons, dont les hôtes intermédiaires sont des Crustacés et les hôtes d'attente des Poissons. Corps de taille moyenne. Tronc inerme cylindroïde. Sous-cuticule contenant de 
nombreux fragments de noyaux géants. Canaux principaux du système lacunaire unis par des anastomoses en réseau à mailles grossièrement polyédriques. Proboscis de taille variable mais toujours cylindroïde, armé de crochets disposés selon des files longitudinales sans dissymétrie dorso-ventrale. Cou très long, cylindroïde, avec souvent un bulbe supérieur donnant insertion au proboscis. Réceptacle à double paroi inséré à la base du proboscis. Ganglion cérébroïde toujours à la partie inférieure du réceptacle. Lemnisci toujours plus courts que le réceptacle, parfois rudimentaires. Organes mâles occupant la moitié postérieure du tronc. Testicules placés l'un derrière l'autre et en contact. Six glandes cémentaires piriformes. Bourse copulatrice avec deux diverticules antérieurs et de nombreux rayons digitiformes. Embryophores ellipsoïdaux à coque mince et lisse, avec grandes hernies polaires de la membrane moyenne.

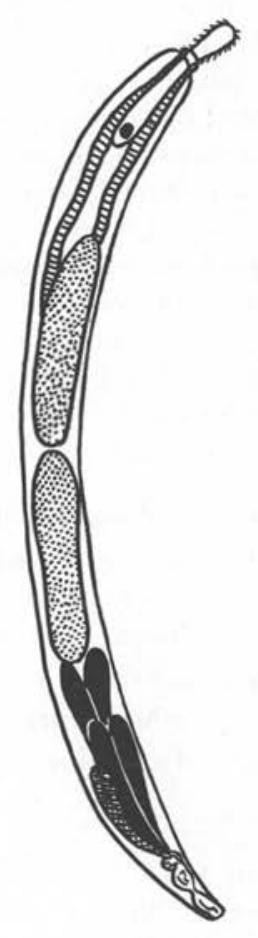

Fig. 30. - Schéma du genre Fissisentis Van Cleave 1931.

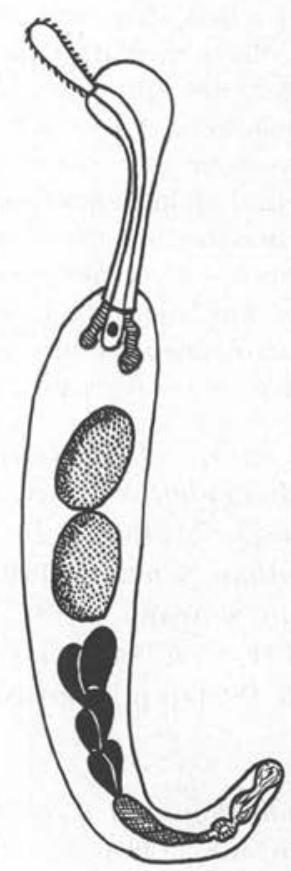

Fig. 31. - Schéma du genre Pomphorhynchus Monticelli 1905.

Genre TyPe : Pomphorhynchus Monticelli 1905.

Cette famille comprend, en outre, les genres : 


\section{Longicollum Yamaguti 1935, \\ Tenuiproboscis Yamaguti 1935.}

\section{Diagnose du genre Pomphorhynchus Monticelli 1905 (=Echinorhynchus (Zoega) O. F. Müller 1776 p.p.)}

Palaeacanthocephala; parasites de Poissons, dont les hôtes intermédiaires sont des Amphipodes et les hôtes d'attente des Poissons. Corps de taille moyenne. Tronc de longueur moyenne, fusiforme, un peu dilaté dans sa partie antérieure. A cette portion fait suite un faux cou, très allongé, cylindroïde, dilaté en bulbe dans sa partie supérieure chez les adultes sexuellement mûrs. C'est sur ce bulbe que s'insèrent le cou et le proboscis. Cet allongement en faux cou de la partie antérieure du tronc permet au parasite d'enfoncer toute la partie antérieure de son tronc à travers la paroi intestinale et le proboscis vient ainsi en contact avec le feuillet viscéral du péritoine de l'hôte. Proboscis cylindroïde, armé de crochets disposés selon des files longitudinales dont la taille décroît régulièrement de l'apex à la base du rostre, les crochets basaux étant, en fait, des épines à racines rudimentaires. Réceptacle inséré à la base du proboscis et très long, s'étendant sur toute la longueur du faux cou pour se terminer dans la partie supérieure du tronc par un renflement terminal dans lequel est placé le ganglion cérébroïde. Lemnisci courts, en massue, à grosse extrémité inférieure. Organes mâles occupant la moitié ou les deux tiers postérieurs de la longueur du tronc. Testicules ovoïdes, l'un derrière l'autre et en contact. Six glandes cémentaires courtes, piriformes. Embryophores ellipsoïdaux, à coque mince, avec grandes hernies polaires de la membrane moyenne.

EsPÈce TYPE: Pomphorhynchus laevis (Zoega) Müller 1776 (= Acanthocephalus lavareti Koelreuther $1771=$ Echinorhynchus laevis (Zoega) Müller $1776=E$. nodulosus Schrank 1790 et 1792 $=E$. dobulaæ Schrank 1790 et $1792=E$. barbi Schrank $1792=$ E. salvenini Schrank $1788=E$. annulatus Gmelin $1791=E$. idbari Gmelin $1791=E$. bramæ Gmelin $1791=$ Echinorhynchus proteus Westrumb 1821 p.p.), parasite de Poissons d'eau douce de l'Ancien Monde.

$2^{\circ}$ Pomphorhynchus tereticollis (Rudolphi 1809) [= Echinorhynchus dobulæ Schrank 1790 p.p. = E. attenuatus O.F. Müller $1776=E$. piscinus Zeder $1800=$ E. longicollis (Pallas) Goeze $1792=$ Echinorhynchus proteus Westrumb 1821 p.p.], parasite de Poissons de mer de l'Ancien Monde.

$3^{\circ}$ Pomphorhynchus bulbocolli (Linkins 1918) Van Cleave 1919, parasite de Poissons d'eau douce d'Amérique du Nord.

$4^{\circ}$ Pomphorhynchus perforator (Von Linstow 1908) (= Echinorhyn- 
chus perforator Von Linstow 1908), parasite de Poissons d'eau douce d'Asie centrale.

$5^{\circ}$ Pomphorhynchus kashmiriensis Kaw 1941, parasite de Poissons d'eau douce du Cachemire.

$6^{\circ}$ Pomphorhynchus dubious Kaw 1941, parasite des Indes, décrit d'après des juvéniles trouvés chez une Grenouille (Rana cyanophlyctis).

$7^{\circ}$ Pomphorhynchus sebastichthydis Yamaguti 1939, parasite de Poissons du Japon.

\section{Diagnose dU GENRE Longicollum Yamaguti 1935} (= Spirorhynchus Harada 1935)

Pomphorhynchida; parasites de Poissons de mer du Pacifique Nord. Corps de taille moyenne. Tronc inerme, cylindroïde, un peu dilaté dans sa partie antérieure. Cou très long, arqué selon sa face ventrale, ou spiralé, fusiforme. Proboscis court, cylindroïde, armé de îles longitudinales de crochets dont la taille diminue progressivement de l'apex à la base du rostre. Les crochets supérieurs ont une racine bien développée de forme simple. Les crochets moyens ont une racine rudimentaire. Les crochets inférieurs ont, à nouveau, une racine bien développée, présentant une longue apophyse supérieure. Réceptacle très long, très mince, traversant tout le cou et se terminant dans la partie supérieure du tronc par une dilatation qui contient le ganglion cérébroïde. Papille sensorielle apicale du rostre bien développée. Lemnisci rudimentaires, réduits à une sorte de collier de lobes sphéroïdaux disposés autour du réceptacle au point où il pénètre dans le tronc. Organes mâles occupant la moitié postérieure de la longueur du tronc. Testicules sphéroïdaux, placés l'un derrière l'autre et en contact. Six glandes cémentaires piriformes. Organe de Saefftigen bien développé. Ovaire allongé, contenu dans le ligament antéro-postérieur. Un seul sphincter vaginal. Orifices génitaux terminaux dans les deux sexes. Embryophores ellipsoïdaux à coque mince, avec grandes hernies polaires de la membrane moyenne.

ESPÈCE TYPE : Longicollum pagrosomi (Yamaguti 1935), provenant de la Mer Intérieure Japonaise. Ce parasite se trouve sous forme de juvéniles chez de nombreux Poissons, mais adulte dans l'intestin d'un seul : Pagrosomus unicolor.

$2^{\circ}$ Longicollum alemniscus (Harada 1935) Fukui et Morishita 1938 (= Spirorhynchus alemniscus Harada 1935), provenant de Formose.

\section{Diagnose DU genre Tenuiproboscis Yamaguti 1935}

Pomphorhynchidæ; parasites de Poissons. Corps de taille moyenne. Tronc fusiforme chez les mâles, cylindroïde chez les femelles, inerme. 
Cou très long, filiforme, cylindroïde. Proboscis très long, en massue faible, à grosse extrémité supérieure, mais surtout très mince, armé de files longitudinales de crochets dont la taille se réduit progressivement de haut en bas du rostre. Tous les crochets possèdent des racines bien développées de forme simple dont la taille se réduit parallèlement à celle de la lame. Réceptacle très long, très mince, traversant le cou et se dilatant au niveau de son fond. Ganglion cérébroïde placé dans cette dilatation terminale du réceptacle. Lemnisci bien développés, en massue, à grosse extrémité inférieure, bien plus courts que le réceptacle, mais, comme ils s'insèrent à la limite du cou et du tronc, leur extrémité inférieure dépasse nettement le fond du réceptacle. Organes mâles occupant les deux tiers postérieurs de la longueur du tronc. Testicules sphéroïdaux placés l'un derrière l'autre et séparés. Six (parfois quatre ?) glandes cémentaires courtes et piriformes. Cloche utérine bien développée. Vagin à deux sphincters, dont seul l'interne est bien développé. Orifices génitaux ter-

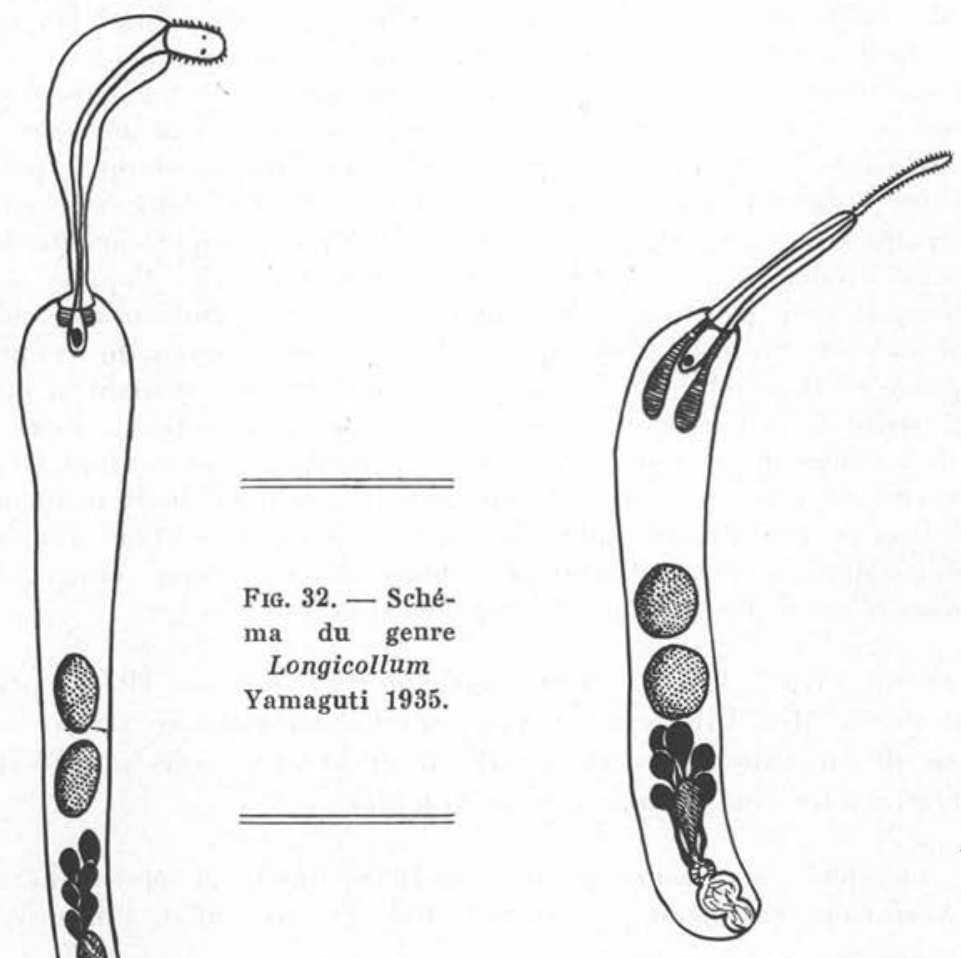

Fig. 33. - Schéma du genre Tenuiproboscis Yamaguti 1935 . 
minaux dans les deux sexes. Embryophores ellipsoïdaux à coque mince et à grandes hernies polaires de la membrane moyenne.

EsPèce TYPE : Tenuiproboscis misgurni Yamaguti 1935, parasite de Poisson d'eau douce du Japon.

$2^{\circ}$ Tenuiproboscis sergenti Choquette et Gayot 1952, trouvé chez un chien errant en Algérie (il s'agit d'un hôte accidentel).

\section{Diagnose de la famille des POLYMORPHIDA Meyer 1931}

Palaeacanthocephala ; parasites, à l'état adulte, d'Oiseaux et de Mammifères aquatiques, dont les hôtes intermédiaires sont des Crustacés et les hôtes d'attente généralement des Poissons, parfois des Batraciens, voire des Reptiles. Corps de taille petite à moyenne. Tronc fusiforme ou cylindroïde, portant des épines cuticulaires sur sa partie antérieure, et parfois possédant des épines génitales. Sous-cuticule contenant de nombreux fragments de noyaux géants. Canaux principaux du système lacunaire latéraux unis par des anastomoses en réseau à mailles grossièrement polyédriques ou régulièrement transversales. Proboscis cylindroïde, assez court, parfois ovoïde, possédant souvęnt de grands crochets spécialisés à la partie moyenne des files ventrales. Réceptacle à double paroi. Ganglion cérébroïde placé à la partie moyenne du réceptacle. Lemnisci généralement plus longs que le réceptacle, souvent aplatis en feuille. Glandes cémentaires dont le nombre varie de deux à six selon les genres. Embryophores caractéristiques d'un cycle aquatique.

Genre type : Polymorphus Lühe 1911.

Cette famille doit être divisée en deux sous-familles :

Corynosominæ Petrotschenko 1956, Polymorphinæ Meyer 1931.

Diagnose DE LA sous-Famille des Corynosominæ Petrotschenko 1956

Polymorphidæ; parasites d'Oiseaux aquatiques ou de Mammifères marins, dont les hôtes intermédiaires sont des Crustacés et les hôtes d'attente des Poissons. Corps de taille moyenne à grande. Tronc souvent hautement modifié, portant toujours un champ ou deux champs d'épines cuticulaires antérieures. Epines génitales parfois présentes, au moins chez les femelles. Proboscis assez court, cylindroïde ou fusiforme, armé de crochets parfois de grande taille à la partie moyenne du rostre, alors que les crochets basaux sont au contraire des épines à racines rudimentaires. Réceptacle à double paroi. Ganglion cérébroïde placé à la partie 
moyenne du réceptacle (au moins chez les Corynosoma). Lemnisci de taille variable, parfois aplatis en feuille. Six ou trois glandes cémentaires selon le genre. Embryophores ellipsoïdaux à coque mince avec grandes hernies polaires de la membrane moyenne.

Genre type : Corynosoma Lühe 1904.

Cette sous-famille comprend, en outre, le genre Bolbosoma Porta 1908 .

\section{Diagnose du genre Corynosoma Lühe 1904}

\section{(= Chentrosoma Monticelli $1905=$ Echinosoma Porta 1907 p.p.)}

Corynosominæ; parasites d'Oiseaux ichthyophages et de Mammifères marins dont les hôtes intermédiaires sont des Crustacés Amphipodes et les hôtes d'attente des Poissons. C'est le seul genre d'Acanthocéphales contenant à la fois des Parasites de Mammifères et d'Oiseaux, et la spécificité pour l'hôte définitif paraît assez étroite, l'espèce n'évoluant pas

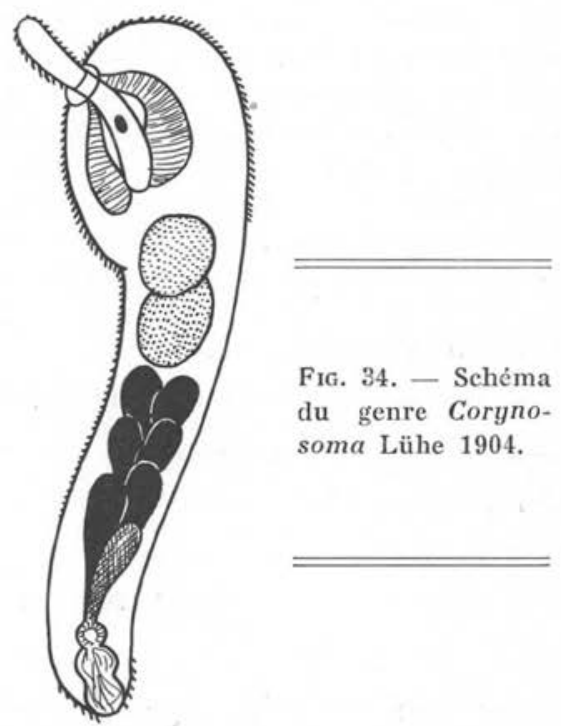

indifféremment jusqu'au stade d'adulte fécond chez un Oiseau ou un Mammifère. Corps de petite taille ou de taille moyenne. Tronc court, en massue, à grosse extrémité antérieure (forme souvent comparée à celle d'une pipe). Cette partie antérieure est souvent dilatée en bulbe ou en disque et porte un champ d'épines cuticulaires triangulaire à pointe inférieure ventrale. Ce champ peut s'étendre ventralement très loin en arrière, arrivant ainsi en continuité avec le champ d'épines, générale- 
ment un peu plus grosses, qui entourent l'orifice génital dans la plupart des espèces, au moins chez les femelles. (Ces épines sont fragiles et sont souvent arrachées lors de la chute du capuchon cémentaire). Proboscis inséré au centre du disque antérieur, à grand axe fortement incliné sur le grand axe du tronc, cylindroïde ou fusiforme, armé de files longitudinales de crochets. Souvent grands crochets placés à la partie moyenne du rostre, au niveau où il est le plus large. Epines à racines rudimentaires à la base des files. Cou court, tronc-conique. Réceptacle assez long. Ganglion cérébroïde placé à la partie moyenne du réceptacle. Lemnisci toujours plus courts que le réceptacle; aplatis en feuille, souvent enroulés latéralement sur eux-mêmes. Organes mâles occupant, soit toute la longueur du tronc, soit seulement sa moitié postérieure. Testicules ovoïdes, placés côte à côte ou l'un derrière l'autre, mais toujours largement en contact. Six glandes cémentaires en longue massue à grosse extrémité supérieure. Embryophores ellipsoïdaux à coque mince, avec grandes hernies polaires de la membrane moyenne. Partie postérieure du tronc souvent invaginée lors de la fixation du fait de la rétraction de muscles très puissants.

EsPÈce TYPE : Corynosoma strumosum (Rudolphi 1802) (=Echinorhynchus strumosus Rudolphi 1802 p.p. $=$ E. histryx Bremser 1824 p.p. $=$ E. ventricosus Rudolphi $1809=$ E. gibbosus Rudolphi 1809 p.p. $=$ E. striatus Villot $1876=$ E. gibber Olsson 1893 p.p. $=$ E. incrassatus (?) Linton $1891=$ E. carchariæ Linton $1891=$ Corynosoma osmeri Fujita $1921=$ C. ambispigerinum Harada 1935), parasite de Phoques de l'Hémisphère Nord, trouvé accidentellement (adultes immatures) chez de nombreux Oiseaux de mer et de nombreux Mammifères, dont les hôtes d'attente sont des Poissons et les hôtes intermédiaires des Crustacés Amphipodes.

$2^{\circ}$ Corynosoma alaskaensis Golvan 1958, parasite d'un Marsouin de l'Alaska.

$3^{\circ}$ Corynosoma australe Johnston 1937, parasite de Phoques d'Australie.

$4^{\circ}$ Corynosoma bullosum (Von Linstow 1892) (=Echinorhynchus bullosum Von Linstow 1892), parasite de Phoques de l'Antarctique.

$5^{\circ}$ Corynosoma cameroni Van Cleave 1953, parasite de Dauphin du Canada.

$6^{\circ}$ Corynosoma cetaceum Johnston et Best 1942, parasite de Cétacés d'Australie.

$7^{\circ}$ Corynosoma curiliensis Gubanov 1942, parasite d'un Cachalot tué dans l'Archipel des Kouriles.

$8^{\circ}$ Corynosoma enhydris Afanas'ev 1941, parasite de la Loutre de mer qui n'est peut-être qu'un synonyme des espèces déjà signalées dans cette région chez Enhydra lutris. 
$9^{\circ}$ Corynosoma falcatum Van Cleave 1953, parasite des Phoques d'Alaska.

$10^{\circ}$ Corynosoma hamanni (Von Linstow 1892 (= Echinorhynchus hamanni Von Linstow $1892=E$. antarcticus Rennie $1906=$ Corynosoma antarcticum Leipner et Atkinson $1914=$ C. hamanni Leipner et Atkinson 1915), parasite de Phoques de l'Antarctique (Géorgie du Sud).

11 ${ }^{\circ}$ Corynosoma obtuscens Lincicome 1943, parasite de Phoques de Californie.

$12^{\circ}$ Corynosoma rauschi Golvan 1958, parasite du Phoque Moine des Midways.

$13^{\circ}$ Corynosoma reductum (Von Linstow 1905) (= Echinorhynchus reductus Von Linstow 1905), parasite de Phoque de l'Arctique.

$1^{\circ}$ Corynosoma semerme (Forssell 1904) (= Echinorhynchus semermis Forssell $1904=$ E. strumosus Rudolphi 1802 p.p. $=$ E. histryx Bremser 1824 p.p. $=E$. gibber Olsson 1893 p.p.), parasite de Phoques de l'Hémisphère Nord, dont les hôtes d'attente sont des Poissons, les hôtes accidentels divers Oiseaux ichthyophages et Mammifères et les hôtes intermédiaires des Crustacés Amphipodes.

$15^{\circ}$ Corynosoma sipho Railliet et Henry 1907, parasite de Phoques de l'Antarctique.

$16^{\circ}$ Corynosoma validum Van Cleave 1953, parasite de Phoques d'Alaska.

17 $7^{\circ}$ Corynosoma villosum Van Cleave 1953, parasite de Phoques d'Alaska.

18 Corynosoma wegeneri Heinze 1934 (= Corynosoma hadweni Van Cleave 1953), parasite de Phoques d'Alaska et du Groenland.

$19^{\circ}$ Corynosoma anatarium Van Cleave 1945, parasite de \& Canards » au Texas.

$20^{\circ}$ Corynosoma clavatum Gosse 1941, parasite de Cormorans d'Australie.

$21^{\circ}$ Corynosoma constrictum Van Cleave 1918, parasite d'Oiseaux ichthyophages d'Amérique du Nord.

$22^{\circ}$ Corynosoma gravida Alegret 1941, parasite de Cormoran du Golfe du Mexique.

$23^{\circ}$ Corynosoma mergi Lundström 1942, parasite d'un Harle de Scandinavie.

$24^{\circ}$ Corynosoma peposacæ (Porta 1914) (= Echinorhynchus peposacæ Porta 1914), parasite de divers Oiseaux ichthyophages du Brésil.

$25^{\circ}$ Corynosoma piriforme (Bremser 1824), parasite trouvé chez un Merle d'Europe Occidentale et dont l'appartenance au genre est fort douteuse, à moins qu'il ne s'agisse d'une infestation accidentelle par une espèce, parasitant normalement un Oiseau d'eau.

$26^{\circ}$ Corynosoma phalacrocoracis Yamaguti 1939, parasite de Cormorans du Japon. 
$27^{\circ}$ Corynosoma tunitæ (Weiss 1914) (Corynosoma histryx tunitæ Weiss 1914), parasite d'un Fou de Bassan tué à Tunis.

$28^{\circ}$ Corynosoma turbidum Van Cleave 1937, parasite de Cormorans d'Afrique du Sud.

Diagnose du genre Bolbosoma Porta 1908

(=Echinorhynchus (Zoega) in O. F. Müller 1776 p.p. $=$ Bolborhynchus Porta $1906=$ Diplospinifer Fukui $1929=$ Pomphorhynchus Leipner et Atkinson 1915).

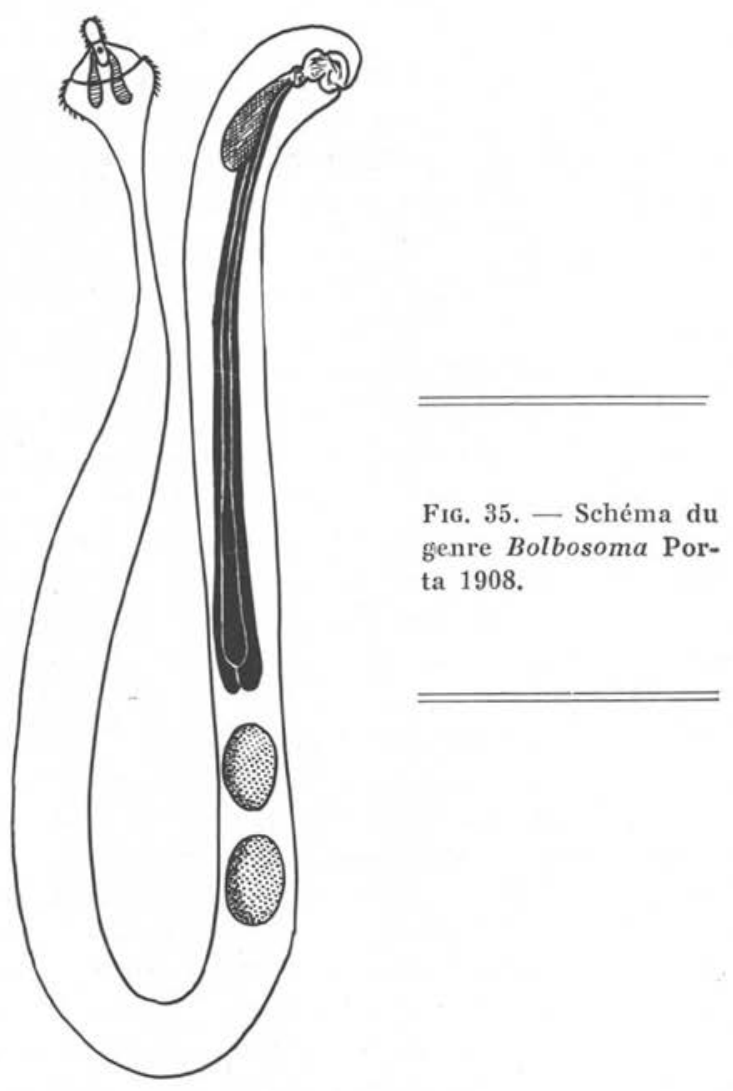

Corynosominæ; parasites de Mammifères marins et, en particulier, de Cétacés, dont les hôtes intermédiaires sont des Crustacés et les hôtes d'attente des Poissons. Corps de taille moyenne à très grande. Tronc long, cylindrique et mince dans toute sa partie postérieure qui représente la partie de beaucoup la plus longue du tronc. La partie toute anté- 
rieure du tronc est dilatée en un bulbe nettement séparé de la partie postérieure par une zone rétrécie plus ou moins longue. Un ou deux champs d'épines cuticulaires sur ce bulbe. Jamais d'épines génitales. Proboscis en courte massue ou conique armé de files longitudinales de crochets dont les basaux sont des épines à racines rudimentaires. Cou court, cylindrique ou tronconique, toujours bien marqué. Place du ganglion cérébroïde inconnue. Lemnisci de taille variable. Organes mâles n'occupant que la partie toute postérieure du tronc. Testicules ellipsoïdaux, placés l'un derrière l'autre, en contact ou très peu séparés. Trois glandes cémentaires très longues. Embryophores ellipsoïdaux à coque mince, avec grandes hernies polaires de la membrane moyenne.

EsPÈCE TYPE : Bolbosoma balenæ (Gmeìn 1790) (= Sipunculus lendix Phipps 1774 p.p. = Echinorhynchus balenæ Gmelin $1790=$ E. porrigens Rudolphi $1819=$ Bolborhynchus porrigens Porta 1906 = Bolbosoma porrigens Porta 1908), parasite de Cétacés.

$2^{\circ}$ Bolbosoma hamiltoni Baylis 1929, parasite de Cétacés de l'Antarctique.

$3^{\circ}$ Bolbosoma heteracanthe (Heitz 1920), trouvé dans les cæca pyloriques d'un Saumon du Rhin (Juvéniles).

$4^{\circ}$ Bolbosoma nipponicum Yamaguti 1939, parasite d'un Cétacé tué au Japon.

$5^{\circ}$ Bolbosoma thunni $(=B$. vasculosum) Harada 1935, trouvé chez un Thon du Japon (Juvéniles).

$6^{\circ}$ Bolbosoma turbinella (Diesing 1851) [= Echinorhynchus turbinella Diesing $1851=E$. balaenocephalus Owen $1803=$ E. ruber Collet 1886 $=$ Bolborhynchus turbinella (Diesing 1851) Porta $1906=$ Bolbosoma turbinella (Diesing 1851) Porta 1908], parasite de Cétacés de l'Antarctique.

$7^{\circ}$ Bolbosoma vasculosum (Rudolphi 1819) [= Echinorhynchus vasculosus Rudolphi $1819=$ E. aurantiacus Risso $1826=$ F. pellucidus Leuckart $1828=E$. annulatus Molin $1858=E$. serrani Linton $1891=E$. bifasciatus Lühe $1904=$ Bolbosoma aurantiacum (Risso 1826) Van Cleave 1924 = Pomphorhynchus turbinella (Diesing) Porta in Leipner et Atkinson 1915], parasite de Cétacés de l'Arctique.

$8^{\circ}$ Bolbosoma brevicolle (Malm 1867) Porta 1908 [= Echinorhynchus brevicollis Malm 1867 = Bolborhynchus brevicollis (Malm 1867) Porta 1906], parasite de Cétacés d'Afrique du Sud.

$9^{\circ}$ Bolbosoma capitatum (Von Linstow 1880) (= Echinorhynchus capitatus Von Linstow 1880), parasite de Cétacés de l'Antarctique.

$10^{\circ}$ Bolbosoma caenoforme (Heitz 1920), parasite trouvé chez un Saumon du Kamtchatka, mais qui, en fait, est peut-être un Corynosoma. 
$11^{\circ}$ Bolbosoma serpenticola (Fukui 1929) (Diplospinifer serpenticola), trouvé chez un Reptile du Japon. L'appartenance au genre Bolbosoma est aussi incertaine que la validité du genre Diplospinifer. Les types devront être réétudiés, mais il sera difficile de conclure puisque ce sont des juvéniles.

$12^{\circ}$ Bolbosoma sp. Van Cleave 1925, trouvé chez un Cétacé du Japon.

$13^{\circ}$ Bolbosoma physeteris Gubanov 1952, parasite de Cétacés de la Mer d'Ochotsh.

$14^{\circ}$ Bolbosoma bobrovoi Krotov et Delaymme 1952, parasite de Phoques de la Mer d'Ochotsh.

\section{Diagnose de la sous-Famille des Polymorphinæ Meyer 1931}

Polymorphidæ; parasites d'Oiseaux aquatiques dont les hôtes d'attente sont des Poissons ou des Batraciens et les hôtes intermédiaires des Crustacés Amphipodes. Corps de taille moyenne. Tronc cylindroïde, dilaté dans sa partie antérieure, laquelle porte un ou deux champs d'épines cuticulaires. Partie antérieure du tronc parfois différenciée en faux cou. Proboscis relativement court, sphéroïdal ou fusiforme, avec parfois des crochets hautement différenciés. Réceptacle à double paroi. Ganglion cérébroïde placé à la partie moyenne du réceptacle. Lemnisci de longueur variable. Organes mâles occupant plus de la moitié postérieure de longueur du tronc. Trois à quatre glandes cémentaires (deux ?) longues et tubuleuses). Jamais d'épines génitales. Embryophores ellipsoïdaux, à coque mince, avec hernies polaires de la membrane moyenne.

Genre type : Polymorphus Lühe 1911.

\section{Diagnose du genre Polymorphus Lühe 1911}

(= Profilicollis Meyer 1931)

Polymorphinæ; parasites du tube digestif des Oiseaux d'eau douce. Corps de taille petite à moyenne. Tronc plus ou moins comprimé, court, fusiforme, jamais très allongé, orné dans sa partie antérieure d'un champ d'épines cuticulaires disposées en quinconce. Canaux principaux du système lacunaire latéraux avec anastomoses en réseau à mailles grossièrement polyédriques. Proboscis court, cylindroïde ou ovoïde, armé de files longitudinales de crochets dont la taille décroît progressivement de l'apex à la base du rostre. Les crochets supérieurs ont des racines bien développées de forme généralement simple, les crochets basaux ont des épines à racines rudimentaires. Cou assez court, tronc-conique et bien marqué. Réceptacle un peu plus long que le proboscis. Ganglion cérébroïde placé à la partie moyenne du réceptacle. Lemnisci digitiformes ou aplatis, aussi longs ou un peu plus longs que le réceptacle. Organes mâles occupant les deux tiers postérieurs de la longueur du tronc. Testi- 
cules sphéroïdaux toujours en contact, placés l'un derrière l'autre ou côte à côte. Trois glandes cémentaires tubuleuses. Orifices génitaux terminaux dans les deux sexes. Embryophores ellipsoïdaux, à coque mince, avec grandes hernies polaires de la membrane moyenne.

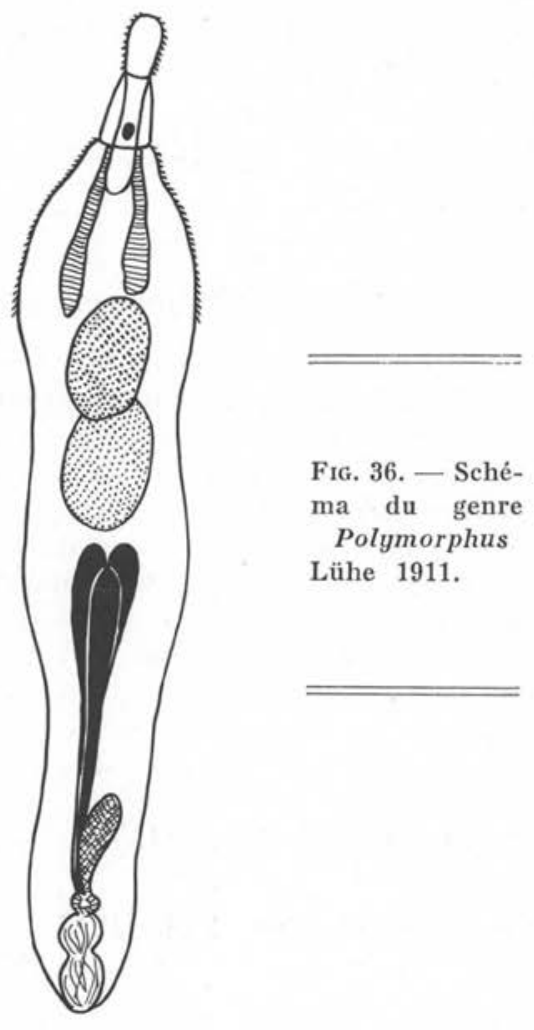

EsPèce TYPE : Polymorphus minutus (Goeze 1782) (=Echinorhynchus minutus Goeze $1782=E$. boschadis Schrank $1788=$ E. anatis Gmelin $1791=E$. collaris Schrank $1792=E$. anatis Froelich $1802=E$. versicolor Rudolphi $1819=E$. polymorphus Bremser $1824=E$. miliarius Zeder 1832), parasite des Canards d'Europe, dont on trouve très souvent les juvéniles chez des Poissons et dont les hôtes intermédiaires sont des Gammares.

$2^{\circ}$ Polymorphus meyeri Lundström 1942, parasite de Canards de Scandinavie.

$3^{\circ}$ Polymorphus miniatus (Von Linstow 1896 (= Echinorhynchus miniatus Von Linstow 1896), parasite de Canards de la Terre de Feu, 
$4^{\circ}$ Polymorphus mutabilis (Rudolphi 1819) Travassos 1926 (=Echinorhynchus mutabilis Rudolphi 1819), parasite d'Oiseaux d'eau du Brésil.

$5^{\circ}$ Polymorphus obtusus Van Cleave 1918, parasite d'Ardéiformes de Floride.

$6^{\circ}$ Polymorphus paucihamatus Heinze 1936, parasite de Mouette, probablement de l'Arctique.

$7^{\circ}$ Polymorphus phippsi Kostylew 1922 (= Sipunculus lendix Phipps $1774=$ Echinorhynchus borealis Gmelin $1791=$ Echinorhynchus anatis mollissima Rudolphi 1809), parasite de l'Eider.

$8^{\circ}$ Polymorphus striatus (Goeze 1782) Lühe 1911 (= Echinorhynchus striatus Goeze $1782=E$. ardex Gmelin $1789=E$. mutabilis Rudolphi 1819), parasite d'Ardéiformes et d'Ansériformes d'Europe.

$9^{\circ}$ Polymorphus strumosoides Lundström 1942, parasite d'Oiseaux de Scandinavie.

$10^{\circ}$ Polymorphus trochus Van Cleave 1945, parasite d'un Foulque d'Amérique du Nord (Ohio).

11 Polymorphus acutis Van Cleave et Starrett 1940, parasite d'un Canard d'Amérique du Nord (Illinois).

$12^{\circ}$ Polymorphus capellæ Yamaguti 1935, parasite d'un Oiseau du Japon.

$13^{\circ}$ Polymorphus contortus (Bremser 1821) Travassos 1926 (= Echinorhynchus contortus Bremser in Westrumb $1821=E$. collurionis Rudolphi 1819). L'appartenance de cette espèce au genre Polymorphus est incertaine, l'espèce est décrite d'après des juvéniles trouvés chez une Pie-grièche d'Europe (Lanius collurio).

$14^{\circ}$ Polymorphus corynoides Skrjabin 1913, parasite de Canards d'Asie Centrale.

15 ${ }^{\circ}$ Polymorpha corynosoma Travassos 1915, parasite d'Ardéiformes du Brésil.

$16^{\circ}$ Polymorphus crassus Van Cleave 1924, parasite d'un Jabiru d'Amérique du Nord.

$17^{\circ}$ Polymorphus cucullatus Van Cleave et Starrett 1940, parasite d'Oiseaux d'Illinois.

$18^{\circ}$ Polymorphus diploinflatus Lundström 1942, parasite de Canards de Scandinavie.

$19^{\circ}$ Polymorphus frontospinosus Tubangui 1935, parasite d'un Héron des Philippines.

$20^{\circ}$ Polymorphus inermis Travassos 1923, parasite d'un Héron du Brésil.

21 ${ }^{\circ}$ Polymorphus kenti Van Cleave 1947, parasite d'une Mouette du Canada.

$22^{\circ}$ Polymorphus magnus Skrjabin 1913, parasite d'Oiseanx aquatiques d'Asie Centrale et d'Europe Orientale. 
$23^{\circ}$ Polymorphus major Lundström 1942, parasite d'Oiseaux aquatiques de Scandinavie.

24. Polymorphus marckii (Porta 1910) (= Corynosoma marchii Porta 1910), parasite d'Oiseaux aquatiques d'Italie.

$25^{\circ}$ Polymorphus marilis Van Cleave 1939, parasite d'Oiseaux aquatiques d'Amérique du Nord (Oklahoma).

$26^{\circ}$ Polymorphus biziuræ Johnston et Edmonds 1948, parasite d'un Canard d'Australie.

Diagnose du sous-genre Profilicollis (Meyer 1931)

(= Falsifilicollis Webster 1948)

Polymorphus à proboscis sphéroïdal, à cou long et étiré.

EsPÈce TYPE : Polymorphus (Profilicollis) botulus (Van Cleave 1916), parasite d'Eider.

$2^{\circ}$ Polymorphus (Profilicollis) arcticus (Van Cleave 1920), parasite d'Eider.

$3^{\circ}$ Polymorphus (Profilicollis) altmani (Perry 1942) Van Cleave 1947 (= Filicollis altmani Perry 1942), parasite d'Oiseaux de Californie.

$4^{\circ}$ Polymorphus (Profilicollis) texensis Webster 1948, parasite d'Oiseaux du Texas.

Nous ne possédons actuellement aucun renseignement sur les genres :

Hexaglandula Petrotschenko 1950,

Skrjabinorhynchus Petrotschenko 1956,

Parafilicollis Petrotschenko 1956,

dont les descriptions ne paraîtront que dans le second tome de la Monographie des Acanthocéphales de Petrotschenko.

Diagnose DU GENRE Arhythmorhynchus Lühe 1911

(= Southwellina Witenberg 1932)

Polymorphinæ; parasites d'Oiseaux ichthyophages, dont les hôtes intermédiaires sont des Crustacés et les hôtes d'attente des Vertébrés aquatiques à sang froid, Poissons ou Batraciens. Corps de taille moyenne. Tronc allongé, cylindroïde, présentant une nette dilatation dans sa partie antérieure. Cette dilatation est assez souvent subdivisée par une striction médiane en deux renflements secondaires. Renflement supérieur portant un ou deux champs d'épines cuticulaires disposées en quinconce, la spinulation couvrant une surface triangulaire à pointe inférieure ven- 
trale. Partie postérieure du tronc parfois renflée en massue. Sous-cuticule contenant de nombreux fragments de noyaux géants, arrondis, et surtout concentrés dans la partie antérieure du tronc, manquant presque totalement en avant et en arrière de cette zone. Proboscis assez long, en fuseau plus ou moins large dans sa partie moyenne. Dissymétrie dorso-

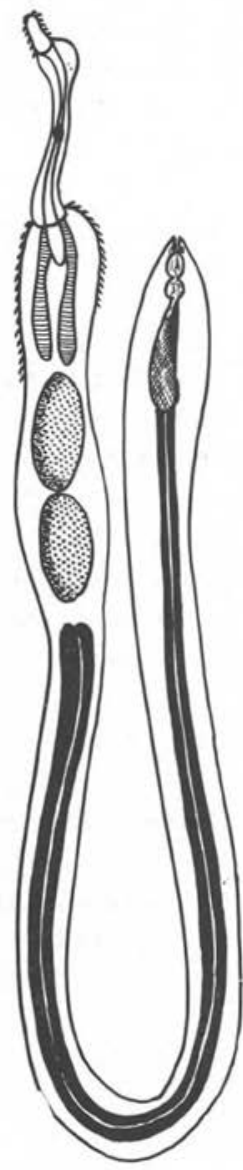

Fig. 37 Schéma du genre Arhythmorhynchus Lühe 1911.

ventrale des crochets plus ou moins accusée, avec parfois présence de grands crochets spécialisés à la partie moyenne des files ventrales. Cou toujours nettement marqué, parfois très long, avec bulbe antérieur sur lequel s'insère le proboscis. Réceptacle long terminé par un renflement placé au-dessous de la limite du cou et du tronc. Ganglion cérébroïde généralement placé à la partie moyenne du réceptacle. Lemnisci toujours longs, dont l'extrémité inférieure dépasse toujours le fond du réceptacle. Organes 
mâles occupant au moins les quatre cinquièmes postérieurs de la longueur du tronc. Testicules dans la dilatation antérieure du tronc, ellipsoïdaux, placés l'un derrière l'autre et un peu séparés. Deux à quatre glandes cémentaires très longues, tubuleuses. Embryophores ellipsoïdaux, à coque mince, avec grandes hernies polaires de la membrane moyenne. Orifices génitaux terminaux dans les deux sexes.

ESPÉCE TYPE : Arhythmorhynchus frassoni (Molin 1858) Lühe 1911 (=Echinorhynchus frassoni Molin $1858=$ E. globicollis Creplin $1829=E$. roseus Molin $1858=E$. rubicundus Molin 1859), parasite d'Oiseaux aquatiques d'Europe.

$2^{\circ}$ Arhythmorhynchus johnstoni nom. nov. [ = Arythmorhynchus frassoni (Molin 1858) in Johnston et Edmonds 1950], parasite d'Oiseaux aquatiques du Queensland.

$3^{\circ}$ Arythmorhynchus longicollis (Villot 1875) (= Echinorhynchus lon. gicollis Villot $1875=E$. invaginabilis Von Linstow $1902=E$. macrourus Bremser $1821=E$. ardeæ purpureæ Rudolphi $1819=$ Arhythmorhynchus anser Florescu 1941), parasite de Mouettes d'Europe.

$4^{\circ}$ Arythmorhynchus longicollis suecica Lundström 1942, parasite d'Oiseaux du littoral scandinave.

$5^{\circ}$ Arythmorhynchus siluricola R.-Ph. Dollfus 1929, décrit d'après des juvéniles trouvés chez un Siluridæe africain indéterminé.

$6^{\circ}$ Arhythmorhynchus plumirostris Van Cleave 1918, parasite d'un Butor d'Amérique du Nord.

$7^{\circ}$ Arythmorhynchus brevis Van Cleave 1916, parasite d'un Butor d'Amérique du Nord.

$8^{\circ}$ Arythmorhynchus comptus Van Cleave et Rausch 1950, parasite de divers Oiseaux d'Amérique du Nord (Alaska).

$9^{\circ}$ Arhythmorhynchus tigrinus Moghe et Das 1953, parasite trouvé sous forme juvénile chez des Grenouilles des Indes et dont les hôtes expérimentaux sont des Corbeaux et des Milans (Moghe et Das 1954) et Das (1953-1954).

$10^{\circ}$ Arhythmorhynchus plicatus (Von Linstow 1883), parasite d'Oiseaux d'Asie Centrale.

11. Arhythmorhynchus trichocephalus (Leuckart in Kaiser 1893), parasite récolté en Floride dont l'hôte est inconnu.

$12^{\circ}$ Arhythmorhynchus eroliæ (Yamaguti 1939) Van Cleave et Rausch 1950 (= Polymorphus eroliæe Yamaguti 1939), parasite d'Oiseaux du Japon.

$13^{\circ}$ Arhythmorhynchus uncinatus (Kaiser 1893) Van Cleave 1924, parasite récolté en Floride dont l'hôte est inconnu.

$14^{\circ}$ Arhythmorhynchus teres Van Cleave 1920, parasite d'une Mouette du Chili. 
$15^{\circ}$ Arhythmorhynchus fuscus Harada 1929, parasite d'un Héron du Japon.

$15^{\circ}$ Arhythmorhynchus hispidus Van Cleave 1925 [ = Arhythmorhynchus fuscus Harada $1929=S$. outhwellina hispida (Van Cleave 1925) Witenberg 1932], parasite de Héron du Japon dont les hôtes d'attente sont des Batraciens.

$16^{\circ}$ Arhythmorhynchus duocinctus Chandler 1935, parasite de Héron d'Amérique du Nord dont les hôtes d'attente sont des Poissons.

$17^{\circ}$ Arhythmorhynchus macracanthus Ward et Winter 1952, décrit d'après des juvéniles trouvés chez des Poissons d'Amérique du Nord.

$18^{\circ}$ Arythmorhynchus distinctus Baer 1956, provenant du Groenland.

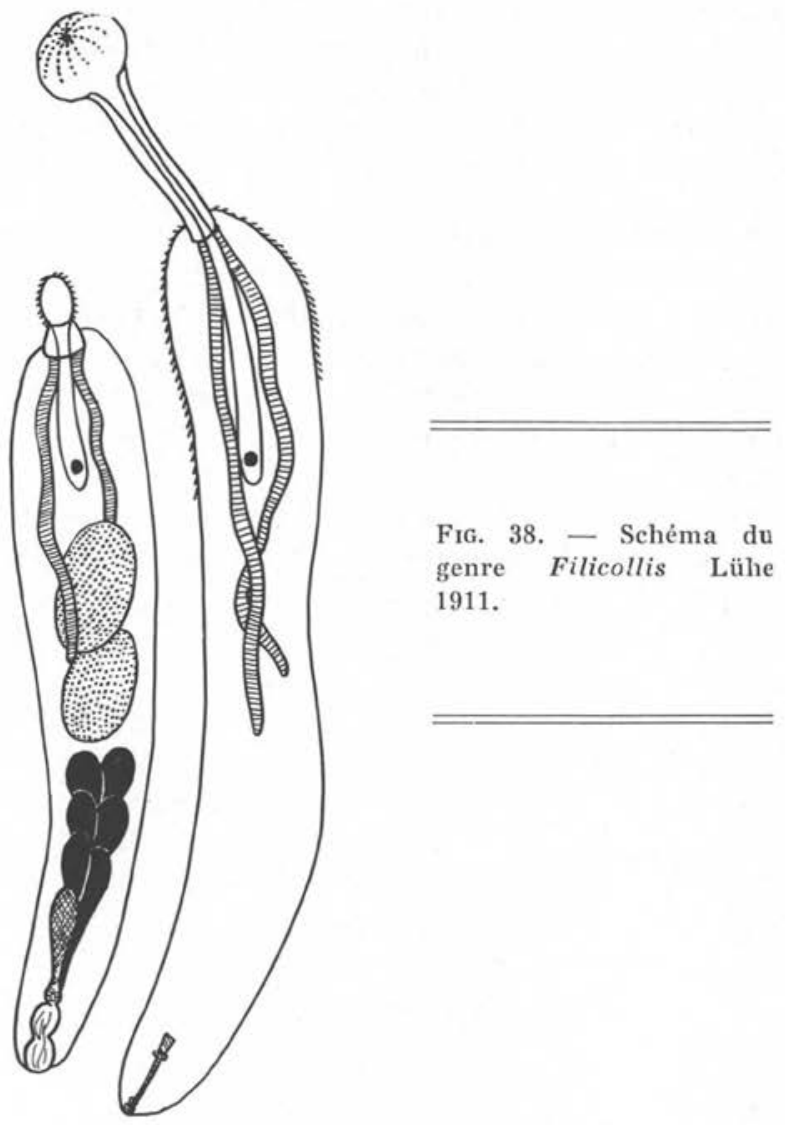

Diagnose du genre Filicollis Lühe 1911

Polymorphinæ; parasites d'Oiseaux aquatiques. Corps de taille moyenne. Tronc plus ou moins aplati, plissé, un peu dilaté en avant, 
mais, dans son ensemble court, trapu. Partie antérieure du tronc couverte, seulement chez les mâles, d'un champ triangulaire à pointe inférieure ventrale, de petites épines cuticulaires disposées en quinconce (c'est le seul exemple d'Acanthocéphales n'ayant conservé leurs épines que dans le sexe mâle. Chez les Filicollis, d'ailleurs, ce sont les femelles qui sont le plus hautement modifiées). Cou toujours bien marqué, parfois très long, cylindrique. Proboscis des mâles court, ovoïde ou sphéroüdal. Proboscis des femelles sphéroïdal, dilaté en bulbe sur lequel sont insérés les crochets. Réceptacle long, s'étendant sur toute la longueur du cou. Ganglion cérébroïde placé dans la partie inférieure du réceptacle. Lemnisci digitiformes, longs. Canaux principaux du système lacunaire latéraux unis par des anastomoses en réseau à mailles grossièrement polyédriques. Organes mâles occupant les deux tiers postérieurs de la longueur du tronc. Testicules sphéroïdaux, placés l'un derrière l'autre, un peu obliquement en contact ou un peu séparés. Six glandes cémentaires allongées, piriformes. Embryophores ellipsoïdaux à coque mince avec hernies polaires de la membrane moyenne.

EsPÈce TYPE : Filicollis anatis (Schrank 1788) (= Echinorhynchus anatis Schrank $1788=E$. alcæ Gmelin $1789=$ E. vesiculosus Schrank $1790=E$. constrictus Zeder $1800=E$. torquatus Froelich $1802=E$. filicollis Rudolphi $1809=E$. polymorphus Bremser 1824 p.p. $=E$. stellaris Molin $1858=E$. laevis von Linstow 1905), parasite d'Oiseaux aquatiques d'Europe.

$2^{\circ}$ Filicollis sphaerocephalus (Bremser 1819) Travassos 1928 (=Echinorhynchus sphaerocephalus Bremser in Rudolphi 1819), parasite d'Oiseaux aquatiques du Brésil.

(à suivre). 